\title{
高等教育国际化背景下仪器分析实验(全英文)教学的实践与体会
}

冯霞 1,2 , 常静 1,2 , 刘俊吉 1,2 , 魏玉萍 $1,2,{ }^{*}$

1 天津大学理学院化学系, 天津 300354

2 天津大学化学化工国家级教学示范中心, 天津 300354

摘要: 就当前高等教育国际化背景下, 结合我校的实际情况, 从教学内容、教材建设、师资队伍培养、授课方式和方法、 考核方式等方面总结了我校开设仪器分析实验(全英文)教学的实践与体会。

关键词: 仪器分析实验; 全英文; 教学方法

中图分类号: G64; O6

\section{Experience on Instrumental Analysis Laboratory Teaching in English for International Students in Tianjin University}

Xia Feng ${ }^{1,2}$, Jing Chang ${ }^{1,2}$, Junji Liu ${ }^{1,2}$, Yuping Wei ${ }^{1,2, *}$

${ }^{1}$ Department of Chemistry, School of Science, Tianjin University, Tianjin 300354, P. R. China.

${ }^{2}$ National Demonstration Center for Experimental Chemistry \& Chemical Engineering Education, Tianjin University, Tianjin 300354, P. R. China.

Abstract: This paper summarized the practice and experience of instrumental analysis laboratory teaching in English in Tianjin University under the background of higher education internationalization. It included aspects of teaching content, selection of teaching materials, teaching staff training, teaching methods and assessment methods.

Key Words: Instrumental analysis laboratory; Teaching in English; Teaching method

高等教育国际化是经济全球化、政治多极化、文化多元化、信息网络化的产物，也是 “双一流” 建设目标下, 高校建设的重要内容 ${ }^{[1]}$ 。留学生教育是大学国际化的重要组成部分, 来华留学生教育 规模已成为衡量高等教育国际化的重要指标 ${ }^{[2]}$ 。2008 年天津大学化工学院化学工程专业通过英国化 学工程师学会(Institution of Chemical Engineers, IChemE)化学工程专业最高级别 “Master Level” 认 证初评, 并于 2014 年通过复评, 成为我国化工学科第一个通过国际专业认证的学科 ${ }^{[3]}$, 标志着我国 化工高等教育在国际化发展进程中取得了突破性进展。配合 IChemE认证, 2015 年我校化工学院化 学工程与工艺专业开始招收国外留学生, 环境学院也相继开始招生, 同时学校进行了相应的课程改 革 ${ }^{[4]}$, 并设置了相关的全英文课程。

仪器分析实验课是为我校化学、化工、材料、环境、药学及相关专业本科生开设的必修基础实 验课, 它是一门实践性很强的基础课, 既独立又需要与仪器分析理论课密切配合。通过仪器分析实 验课的学习, 使学生进一步加深理解仪器分析基本原理, 掌握常用仪器的基本操作, 提高学生对理 论知识的应用能力。仪器分析实验(全英文)是留学生第一学年完成化学分析实验(全英文)课程的学

收稿: 2019-07-24; 录用: 2019-08-20; 网络发表: 2019-09-27

“通讯作者, Email: ypwei@tju.edu.cn

基金资助：国家自然科学基金(51403151); 天津大学教学改革项目(TS14D056) 
习, 掌握了分析化学基本操作后, 在第三学年与仪器分析理论课(全英文)同步开设的一门必修基础 实验课。目前已为两届留学生开设。本文将从教学内容、教材建设、师资培养、授课方式和方法、 考核方式等几个方面取得的初步经验进行介绍。

\section{1 教学内容}

仪器分析是以物质的物理和物理化学性质为基础建立的一种分析方法。学生通过仪器分析实验, 利用仪器对物质进行定性、定量以及形态分析, 从而加深对仪器构造以及分析原理理论知识的理解。 由于留学生来自不同的国家和地区, 学生专业基础参差不齐且普遍较差, 化学实验能力差异也较大, 同时兼顾学校有限的仪器分析教学资源。目前仪器分析实验(全英文)课程计划总学时为 24 学时, 内 容涉及原子吸收光谱、紫外-可见光谱、气相色谱以及电化学等分析方法。目前共开设 6 个实验 4 学 时(实验), 分别是 “离子选择性电极法测定水中氟含量” “邻二氮菲分光光度法测定微量铁” “原子吸 收法测定废液中铜的含量” “苯酚类化合物的紫外吸收光谱” “恒电流电解法测定铜含量” “醇系物的 气相色谱分析”，对应的英文内容如表 1 所示。教学安排上尽可能将现有的在校本科生实验全覆盖, 保证学时的同时适当降低难度。除 “离子选择性电极法测定水中氟含量” 实验, 留学生只做标准曲 线法; 而在校本科生须同时利用标准曲线法和标准加入法两种方法进行测试; 其他 5 个实验内容与 要求与在校本科生基本一致(表 2)。

\section{表 1 仪器分析实验(全英文)实验内容和学时分配}

\begin{tabular}{c}
\hline 实验内容(中英文) \\
\hline 离子选择性电极法测定水中氟含量(Determination of fluoride in tap water with ion selective electrodes) \\
邻二氮菲分光光度法测定微量铁(Spectrophotometric determination of iron using 1,10-phenanthroline) \\
原子吸收法测定废液中铜的含量(Atomic absorption spectroscopy analysis of trace copper) \\
苯酚类化合物的紫外吸收光谱(Ultraviolet-visible spectroscopy of phenols) \\
恒电流电解法测定铜含量(Electrogravimetric determination of copper in $\left.\mathrm{CuSO}_{4} \cdot 5 \mathrm{H}_{2} \mathrm{O}\right)$ \\
醇系物的气相色谱分析(Gas chromatography analysis of alcohol, propanol and butanol)
\end{tabular}

表 2 本科生和留学生仪器分析实验安排和教学要求

\begin{tabular}{lll}
\hline \multicolumn{1}{c}{ 实验安排 } & \multicolumn{1}{c}{ 本科生教学要求 } & \multicolumn{1}{c}{ 留学生教学要求 } \\
\hline 离子选择性电极法测定水中氟含量 & 标准曲线法 + 标准加入法测定自来水中氟含量 & 标准曲线法测定自来水中氟含量 \\
邻二氮菲分光光度法测定微量铁 & 吸收曲线绘制 + 标准曲线法定量测试 & 吸收曲线绘制 + 标准曲线法定量测试 \\
原子吸收法测定废液中铜的含量 & 标准曲线法定量测试 & 标准曲线法定量测试 \\
苯酚类化合物的紫外吸收光谱 & $\mathrm{pH}$ 及对硝基取代基对苯酚紫外光谱的影响 & $\mathrm{pH}$ 及对硝基取代基对苯酚紫外光谱的影响 \\
恒电流电解法测定铜含量 & 电解前后铂电极重量的变化计算铜含量 & 电解前后铂电极重量的变化计算中铜含量 \\
醇系物的气相色谱分析 & 面积归一法进行醇系物定量分析 & 面积归一法进行醇系物定量分析 \\
\hline
\end{tabular}

\section{2 教材建设}

仪器分析实验受实验室资源和设备条件制约, 开设实验须依托现有设备展开。目前仪器分析实 验(全英文)开设的 6 个实验与在校本科生的实验内容基本一致。在教材选用上, 以我校编写的《基 础化学实验教程》教材 ${ }^{[5]}$ 相应内容的英文翻译版为课程教材, 以英文原版《Chemistry Experiments for Instrumental Methods》作为教师的参考用书 ${ }^{[6]}$ 。实验指导教师将 6 个实验内容翻译成英文, 并做成相 应的 PPT, 课前将 PPT 和相应的实验讲义发给学生, 实验课上再利用多媒体讲述实验内容。经过两 年的教学实践, 取得了较为满意的教学效果。 


\section{3 师资培养}

全英文教学需要具有专业理论知识、有留学背景且英文表达能力比较好的教师担任主讲。为了 配合全英文教学, 提高英文授课的能力和技巧, 我校设置了专门的国际化教学学术型师资培训项目, 采取 “请进来” 和 “送出去” 相结合的培训模式 $\left[{ }^{[7]}\right.$ 。“请进来” 是指与国外教师培训机构合作, 将欧 美高校教师 “请” 进天津大学。从 2013 年开始我校每年暑期组织 40 余名负责全英文授课的教师参 加英文教学(Teaching in English, TIE)项目培训, 内容覆盖英语教学法、教师核心英语口语、美国高 等教育、学术英语写作技巧以及创新实验教学等方面。“送出去” 是将我校教师 “送” 到国外高校 进修, 亲身参与到国外高校的教学与管理。2015 年开始我校每年选拔 10 名左右教师赴美国加州大 学洛杉矶分校参加为期六个月的进修学习, 培训期间要求教师完成英语教学法培训, 选修一门专业 课程并通过考试。该类培训项目一方面提高了教师英语的听说能力, 同时也学到了国外教师的授课 方法与技巧, 提升了本科生教学特别是留学生的教学水平。

\section{4 授课方式、方法}

\section{1 “引导探索、激发兴趣”式教学方法}

结合多媒体, 实验课前从回顾理论知识和实验原理, 逐渐过渡到实验内容中, 同时引导学生建 立相应的联系。举例来说, “离子选择性电极法测定水中氟含量” 实验与日常生活非常贴近, 在实验 前讲述较多的背景知识, 如龊齿的成因以及氟含量高低对人体健康的影响, 去激发学生的实验兴趣。 再比如 “邻二氮菲分光光度法测定微量铁” 实验, 该实验通过测定邻二氮菲与二价铁形成的配合物 的吸光度, 进而测定铁含量。该实验的方法也可用于天然气中硫化氢的测定 ${ }^{[8]}$, 通过实际生活的具 体应用, 引导学生探索的同时加深理解。另外, “恒电流电解法测定铜含量” 和 “原子吸收法测定废 液中铜的含量” 这两个实验都是测定铜含量, 其中电解法测铜通常用于测定合金中铜含量, 而原子 吸收法通常用于微量和痕量分析, 如药物或食品中的重金属含量的检测等。同时告诉学生为了保证 数据的准确性, 电解法测铜实验结束后再将电解液进行原子吸收测定, 两部分铜的测量结果加和才 是最终准确的铜含量。

\section{2 不同的师生比授课}

“离子选择性电极法测定水中氟含量” 和 “邻二氮菲分光光度法测定微量铁” 这两个实验, 由 于仪器基本能保证一人一组, 采用大班授课。而对于 “原子吸收法测定废液中铜的含量” “有机化合 物的紫外吸收光谱测定” “恒电流电解法测定铜含量” 和 “醇系物的气相色谱分析” 4 个实验, 因为 大型仪器资源有限, 目前约按师生比 1:15 的小班授课, 目的是每个学生都有机会操作仪器。

\section{3 “点对点” 的方式解决问题}

学生在实验过程中, 指导教师须密切观察、逐一检查发现每位学生在仪器使用过程中出现的问 题, 使用英语口头沟通和交流, 及时纠正不当的操作, 让学生意识到自己存在的问题并改正。因为 留学生专业基础知识薄弱, 实验中涉及的操作及原理知识科技词汇较多, 学生在短时间内掌握相对 较为困难。指导教师除了对仪器的理论和原理知识、仪器构造、操作步骤和注意事项进行较详尽的 讲解外, 必要时, 会重复讲述两遍甚至三遍。同时在课程安排上, 仪器分析实验(全英文)的实验通常 安排在仪器分析(全英文)理论课相应的内容之后, 且课前要求学生必须提前预习。两届的留学生仪 器分析实验课(全英文)的实践告诉我们, 激发兴趣是第一步; 更多的时间用在不停巡视发现问题并 英语口头沟通 “点对点” 解决问题上。实验结束后, 学生将原始数据交给实验指导教师批阅, 一方 面可以通过原始数据发现实验中存在的问题, 以便及时沟通解决; 同时引导学生掌握如何有效归纳、 总结实验原始数据, 为实验报告科学地提供简洁、准确、真实有效的数据。

\section{5 考核方式}

仪器分析实验(全英文)课程的目标和特点决定了学生不仅需要了解常用仪器结构的基本原理、 
仪器主要组成部件及其工作过程, 掌握常用仪器的基本操作, 学会合理地选择实验条件, 提高动手 能力和实验技能, 同时还需要培养他们良好的科技英语表达能力, 熟练利用英文撰写实验报告, 正 确处理数据和表达实验结果。因此, 仪器分析实验(全英文)课程成绩评定以实验操作、实验能力、实 验结果及实验报告是否准确、是否规范化为主要依据, 采取平时成绩为主的办法。教学中我们发现, 留学生两级分化严重, 部分留学生学习积极性较高且态度端正, 实验报告中附有详细的实验结果、 讨论及误差分析等; 部分留学生纪律性差, 上课迟到甚至缺席。因此在成绩评定中, 实验课出勤占 $40 \%$, 实验操作占 $40 \%$, 实验报告占 $20 \%$ 。鼓励学生在实验中有所创新, 对于有独特见解的学生, 成绩可以从优评定。

\section{6 结语}

经过两届留学生的教学实践, 我们初步构建了仪器分析实验(全英文)课程的教学体系, 即以在 校本科生的仪器分析实验课程作为基本教学内容, 以自编英文版讲义为教材, 辅以英文原版教材为 参考书。授课方式上采用 “引导探索-激发兴趣” 式教学模式, 不同的师生比授课方式, 实验过程中 通过 “点对点” 的方式及时沟通解决实验中遇到的问题, 提高英语口语表达能力的同时达到了实验 的目的。此外, 对于实验报告的书写, 培养了其正确处理数据和表达实验结果的能力。目前看来, 教学效果良好。然而, 由于时间有限, 仪器分析实验(全英文)课程的教学体系还需要进一步拓展和优 化, 以适应国际化教育教学体系的需求。

参 考 文 献

[1] 刘强, 康云菲. 高校教育管理, 2018, 12 (5), 23.

[2] 陆菁, 凌慧, 潘修扬. 中国高教研究, 2019, No. 1, 22.

[3] 辛峰, 徐心茹. 化工高等教育, 2016, 33 (4), 15 .

[4] 孙津生, 吕影. 化工高等教育, 2019, No. 1, 28.

[5] 唐向阳, 余莉萍, 朱莉娜, 高洪苓, 陶敏莉. 基础化学实验教程. 第4版. 北京: 科学出版社, 2015.

[6] Sawyer, D. T.; Heineman, W. R.; Beebe, J. M. Chemistry Experiments for Instrumental Methods; Wiley: New York, 1984.

[7] 刘洁, 靳楠, 王东华, 丁伯慧. 天津大学学报(社会科学版), 2018, 20 (6), 566.

[8] Tekin, T.; Boyabat, N.; Bayramoğlu, M. Int. J. Chem. Kinet. 1999, 31, 331. 